\title{
Recent statistics on lightning fatalities in Malaysia
}

\begin{abstract}
It is a fact that Malaysia encounters more than $70 \%$ of power outages due to lightning and it is known as the ñCrown of Lightningò in the world. The effects of lightning on electrical/communication networks and structures account for equipment damage, downtime/data losses and malfunctioning of control and automated systems that may cost the nation over RM 250 million and thousands of human injuries and deaths. Good statistics about death and injuries around the world are just beginning to be collected. This paper presents an update of the statistics on lightning fatalities in Malaysia and some comparisons with other countries around the world. In addition, a case study that accounts the geographical location, lightning detection data and distribution system layout will be presented.
\end{abstract}

Keyword: Lightning; Lightning injury; Lightning fatalities; Lightning detection system 